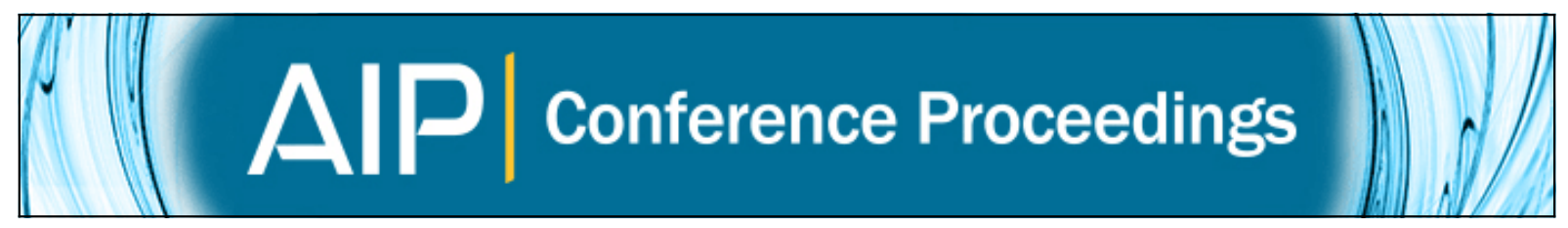

\title{
Magnesium Isotopes in Halo Stars
}

Jorge Meléndez and Judith G. Cohen

Citation: AIP Conference Proceedings 990, 181 (2008); doi: 10.1063/1.2905536

View online: http://dx.doi.org/10.1063/1.2905536

View Table of Contents: http://scitation.aip.org/content/aip/proceeding/aipcp/990?ver=pdfcov

Published by the AIP Publishing

Articles you may be interested in

Analysis of Two Carbon Stars in the Carina Dwarf Spheroidal Galaxy

AIP Conf. Proc. 1001, 205 (2008); 10.1063/1.2916965

Understanding the Characteristics of EMP Stars as Probes for the Early Universe: Stellar Evolution of Low- and Intermediate-Mass EMP Stars

AIP Conf. Proc. 990, 276 (2008); 10.1063/1.2905563

Abundance Ratios in Carbon-Enhanced Metal-Poor Stars and the Intermediate-Mass Star Initial Mass Function AIP Conf. Proc. 990, 197 (2008); 10.1063/1.2905540

Metallicity Discrepancy between RGB and RHB Stars of the Globular Cluster M15

AIP Conf. Proc. 990, 184 (2008); 10.1063/1.2905537

Stellar Nucleosynthesis in the Galactic History: the Carbon Stars

AIP Conf. Proc. 990, 178 (2008); 10.1063/1.2905535 


\title{
Magnesium Isotopes in Halo Stars
}

\author{
Jorge Meléndez* and Judith G. Cohen ${ }^{\dagger}$ \\ ${ }^{*}$ Research School of Astronomy \& Astrophysics, Mt. Stromlo Observatory, Cotter Rd., Weston Creek, ACT 2611, \\ Australia \\ †Palomar Observatory, California Institute of Technology, Pasadena, CA 91125
}

\begin{abstract}
We have determined Mg isotope ratios in halo field dwarfs and giants in the globular cluster M71 based on high $\mathrm{S} / \mathrm{N}$ high spectral resolution $\left(R=10^{5}\right)$ Keck HIRES spectra. Unlike previous claims of an important contribution from intermediate-mass AGB stars to the Galactic halo, we find that our ${ }^{26} \mathrm{Mg} /{ }^{24} \mathrm{Mg}$ ratios can be explained by massive stars.
\end{abstract}

Keywords: halo stars; isotopic abundances; globular clusters: M71

PACS: 97.10.Tk; 98.20.Gm; 98.35.Bd; 98.35.Gi

\section{INTRODUCTION}

Magnesium is composed of three stable isotopes ${ }^{24} \mathrm{Mg}$, ${ }^{25} \mathrm{Mg}$ and ${ }^{26} \mathrm{Mg}$, which can be formed in massive stars (e.g., [1]). The lightest isotope is formed as a primary isotope from $\mathrm{H}$, while ${ }^{25,26} \mathrm{Mg}$ are formed as secondary isotopes. The heaviest $\mathrm{Mg}$ isotopes are also produced in intermediate-mass AGB stars [2], so the isotopic ratios ${ }^{25,26} \mathrm{Mg} /{ }^{24} \mathrm{Mg}$ increase with the onset of AGB stars. Therefore, $\mathrm{Mg}$ isotopic ratios in halo stars could be used to constrain the rise of AGB stars in our Galaxy.

It is important to know when AGB stars begin to enrich the halo in order to disentangle the contribution of elements produced by intermediate-mass stars from those produced by massive stars. For example, the high nitrogen abundances observed in metal-poor stars can be explained by fast-rotating massive stars (e.g., [3]) or alternatively by intermediate-mass stars, although the latter option may be unlikely because those stars may not have had time to enrich the halo due to their longer lifetime.

The study of $\mathrm{Mg}$ isotope ratios is also important to understand the abundance variations in globular clusters (e.g., $[4,5])$. Mg isotopic abundances can be obtained from the analysis of $\mathrm{MgH}$ lines in cool stars (e.g., [6, $7,8,9,10,11,12,13])$. In this work, we determine $\mathrm{Mg}$ isotopic ratios in cool halo dwarfs and giants in the globular cluster M71 employing high resolution high S/N spectra taken at Keck.

\section{MG ISOTOPES IN HALO DWARFS}

Three cool halo dwarfs were observed at Keck I employing the upgraded HIRES [14] which provides now a maximum resolving power of $R \approx 10^{5}$. For details see [13].

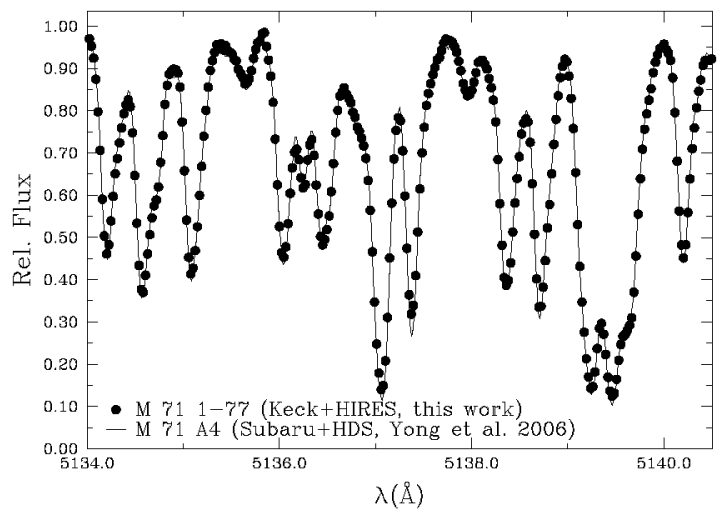

FIGURE 1. Comparison of M71 1-77 observed with Keck+HIRES (circles, this work) and M71 A4 observed with Subaru + HDS (line, [4]). Both stars have similar stellar parameters.

The isotopic ratios were obtained from three wavelength regions at $5134.6,5138.7$ and $5140.2 \AA$ (e.g., $[10,12])$. Isotope ratios were obtained by spectral synthesis using a new line list including atomic lines, $\mathrm{C}_{2}$ and $\mathrm{MgH}$ lines [13]. Macroturbulence was estimated using the Ni I 5115.4 $\AA$ and Ti I $5145.5 \AA$ lines, and other lines around $5585 \AA$.

Low ${ }^{25,26} \mathrm{Mg} / \mathrm{Mg}$ ratios were obtained [13], in agreement with bona-fide halo dwarfs from [12]. These low ratios are in good agreement with yields of massive stars (e.g., $[15,16])$.

\section{MG ISOTOPES IN M71 GIANTS}

Five giants in the globular cluster M71 $([\mathrm{Fe} / \mathrm{H}]=-0.7$, [17]) were observed with HIRES at $R=10^{5}$. We present the analysis of three of them and also of M71 A4 ob- 
tained with the HDS at Subaru by [4]. The latter reduced spectrum was kindly made available to us by D. Yong and W. Aoki.

In Figure 1 we compare our M71 1-77 Keck spectrum with the Subaru spectrum of M71 A4 [4]. As it can be seen, even though the spectra are of two different stars (although of similar stellar parameters), the similarity is very impressive, showing that both data reductions are in excellent agreement.

The atmospheric parameters have been determined as in [18]. Iron lines were used to estimate the microturbulence, $[\mathrm{Fe} / \mathrm{H}]$ and to check the stellar parameters. The iron lines were carefully selected in order to avoid blends by atomic and $\mathrm{CN}$ lines. $\mathrm{CN}$ blends were visually inspected by comparing a synthetic spectrum computed with laboratory $\mathrm{CN}$ lines (e.g., $[19,20]$ ) with the high resolution visible atlas of the cool giant Arcturus [21].

Reliable laboratory oscillators strengths are not available for a large fraction of the FeI lines, so the lines with accurate oscillator strengths were used to provide the zero point of astrophysical $g f$-values. The oscillator strengths for FeII lines are from the laboratory normalization performed by [24].

A good determination of the stellar intrinsic broadening is necessary for a reliable determination of $\mathrm{Mg}$ isotope ratios. The intrinsic broadening is due to both rotation and macroturbulence (e.g., [22]), but in old metalpoor stars we expect the intrinsic broadening to be mostly due to macroturbulence. In these cool metal-rich giants the usual diagnostics for macroturbulence (Ni I 5115.4 and Ti I $5145.5 \AA$ ) seem blended so other lines were used for the determination of the macroturbulence velocity.

As for the field dwarfs, the isotope ratios in giants were determined from three regions, except that in our cool giants the $5134.6 \AA$ feature seems blended, so instead we use the $5134.3 \AA$ feature. A $\chi^{2}$ fit for the $5140.2 \AA$ region is shown in Figure 2.

Our Mg isotope ratios are shown in Figure 3, where a comparison with models [16] is also shown. Our data favors massive stars instead of intermediate-mass AGB stars even at the high metallicity of $\mathrm{M} 71([\mathrm{Fe} / \mathrm{H}]=$ $-0.7)$.

\section{O, NA, MG AND AL IN M71 GIANTS}

We have also determined abundances of $\mathrm{O}, \mathrm{Na}, \mathrm{Mg}$ and $\mathrm{Al}$ in M71 giants. The abundances were determined by both equivalent widths and spectral synthesis.

Unlike other clusters that show large abundance variations (e.g., [23]), the four giants in M71 have essentially identical $\mathrm{O}, \mathrm{Na}, \mathrm{Mg}$ and $\mathrm{Al}$ abundances. Note that the $\mathrm{Mg}$ isotope ratios in these four giants is also constant within the errors (Figure 3). High resolution observations of a larger number of M71 giants will be important in order
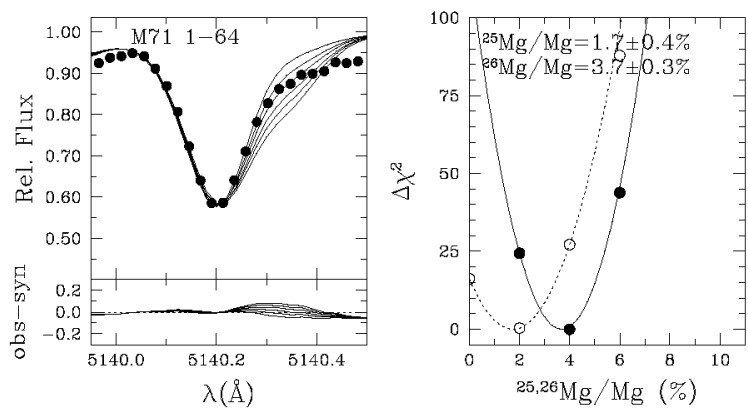

FIGURE 2. Fits for the $5140.2 \AA$ region in the giant M71 1-64. Observed spectra are represented with filled circles, and synthetic spectra with solid lines. The calculations were performed for ${ }^{25,26} \mathrm{Mg} / \mathrm{Mg}$ ratios of $0,2, \ldots 10 \%$. The relative variation of the $\chi^{2}$ fits are shown as a function of the isotopic abundance.

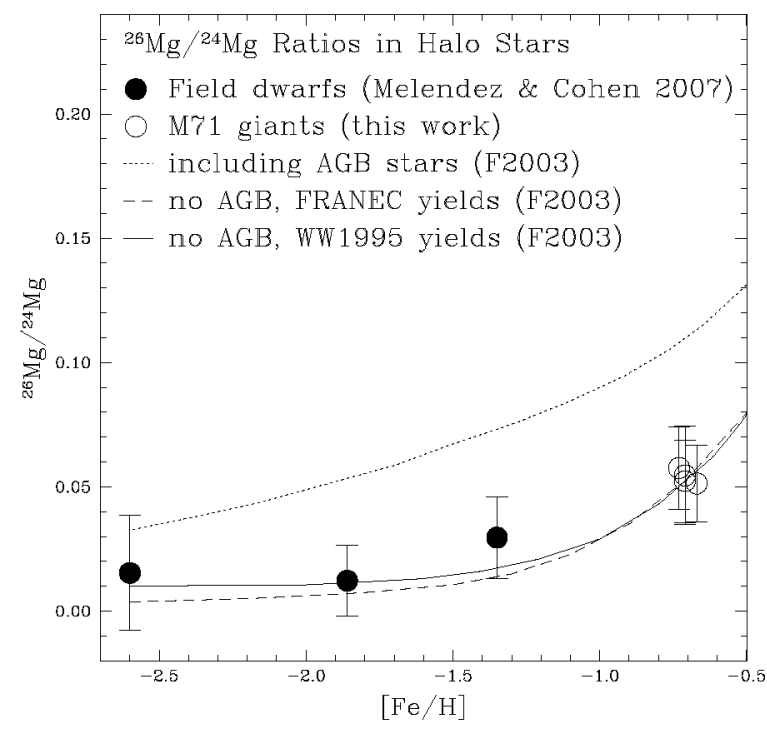

FIGURE 3. ${ }^{26} \mathrm{MgH}^{24} \mathrm{MgH}$ as a function of $[\mathrm{Fe} / \mathrm{H}]$ in halo dwarfs (filled circles) and M71 giants (open circles). Models including massive stars and intermediate-mass AGB stars ([16], F2003) are also shown. The no AGB model agrees better with the observed data

to determine how homogeneous this cluster is.

The oxygen abundance of M71 giants seems undepleted, and consistent with the constant $[\mathrm{O} / \mathrm{Fe}]$ ratio for halo stars found by $[24,25]$, in the broad metallicity range $-3.2<[\mathrm{Fe} / \mathrm{H}]<-0.4$. 


\section{CONCLUSIONS}

Our ${ }^{26} \mathrm{Mg} /{ }^{24} \mathrm{Mg}$ ratios in both field dwarfs and $\mathrm{M} 71$ giants can be explained by massive stars (e.g., [16]). Even at the high metallicity of M71 $([\mathrm{Fe} / \mathrm{H}]=-0.7)$ there is no need to invoke an important contribution from intermediate-mass AGB stars.

We plan to obtain more high resolution high $\mathrm{S} / \mathrm{N}$ HIRES spectra of more field halo dwarfs and M71 giants.

\section{ACKNOWLEDGMENTS}

We thank D. Yong and W. Aoki for kindly providing a Subaru spectrum of M71 A4. JM acknowledges partial support from the Australian Research Council to Martin Asplund. JGC is grateful for partial support to NSF grant AST-0507219.

\section{REFERENCES}

1. S. E. Woosley and T. A. Weaver, ApJS 101, 181-235 (1995).

2. A. I. Karakas and J. C. Lattanzio, PASA 20, 279-293 (2003).

3. C. Chiappini et al., $A \& A 449$, L27-L30 (2006).

4. D. Yong, W. Aoki, and D. L. Lambert, ApJ 638, 1018-1027 (2006).

5. M. Prantzos, C. Charbonnel, and C. Iliadis, $A \& A, \mathbf{4 7 0}$, 179-190 (2007).

6. A. M. Boesgaard, $A p J$ 154, 185-190 (1968).

7. J. Tomkin and D. L. Lambert, $A p J$ 235, 925-938 (1980).

8. B. Barbuy, $A \& A$ 151, 189-197 (1985).

9. B. Barbuy, $A \& A$ 172, 251-256 (1987).

10. A. McWilliam and D. L. Lambert, MNRAS 230, 573-585 (1988).

11. P. L. Gay and D. L. Lambert, $A p J \mathbf{5 3 3}, 260-270$ (2000).

12. D. Yong, D. L. Lambert, and I. I. Ivans, $A p J 599$, 1357-1371 (2003).

13. J. Meléndez and J. G. Cohen, $A p J$ 659, L25-L28 (2007).

14. S. S. Vogt et al., Proc. SPIE 2198, 362-375 (1994).

15. A. Goswami and N. Prantzos, $A \& A$ 359, 191-212 (2000).

16. Y. Fenner et al., PASA 20, 340-344 (2003).

17. S. .V. Ramírez, J. G. Cohen, J. Buss, and M. M. Briley $A J$ 122, 1429-1437 (2001).

18. J. G. Cohen, B. B. Behr, and M. M. Briley, $A J \mathbf{1 2 2}$, 1420-1428 (2001).

19. J. Meléndez and B. Barbuy, ApJS 124, 527-546 (1999).

20. P. Coelho, B. Barbuy, J. Meléndez, R. P. Schiavon, and B. V. Castilho, $A \& A$ 443, 735-746 (2005).

21. K. Hinkle, L. Wallace, J. Valenti, and D. Harmer, Visible and Near Infrared Atlas of the Arcturus Spectrum 3727-9300 A, ASP, San Francisco, (2000).

22. S. Hekker and J. Meléndez, $A \& A$ 475, $1003-1009$ (2007).

23. J. G. Cohen and J. Meléndez, $A J$ 129, 303-329 (2005).

24. J. Meléndez, N. G. Shchukina, I. E. Vasiljeva, and I. Ramírez, ApJ 642, 1082-1097 (2006).
25. I. Ramírez, C. Allende Prieto, and D. Lambert $A \& A 465$, 271-289 (2007). 